\title{
The effect of dry needling in basketball players analyzed using thermographic cameras
}

\author{
Barrios Pitarque C. ${ }^{1} \quad$ Yeste Fabregat M. ${ }^{1}$ \\ ${ }^{1}$ Escuela de Doctorado, Universidad Católica de Valencia, Valencia, Spain \\ Rev Fisioter Invasiva 2019;2:128-129.
}

\begin{abstract}
Keywords

- thermography

- dry needling

- myofascial trigger points

- efficacy
\end{abstract}

Background Approximately $30 \%$ of patients attend primary care consultations for pain, in which the presence of myofascial pain syndrome (MPS) has been confirmed, provoked by myofascial trigger points (MTrPs). Unfortunately, the treatment of this syndrome is resistant to medication and entails an erroneous diagnosis. Novel methods such as sonoelastography and elastography using magnetic resonance, have recently enabled non-invasive images of trigger points. However, both are costly and difficult to access. Therefore, the identification of MTrPs is still based on the palpable diagnosis criteria defined by Travell and Simons. Interestingly, MPS has been defined as nociceptive pain, however, currently the sympathetic nervous system (SNS) is considered increasingly important for the widespread pain related to MPS and this is indicated with greater frequency. The use of the infrared camera is accepted as an objective method for the diagnosis of patients with pain, especially if activity of the SNS is involved. Thermography has high reliability for muscle exam. Hyperthermic images appear when inflammatory reactions are present, increasing the blood flow due to a greater cell activation. In contrast, hypothermic activation is found when there is compression or degenerative processes. Dry needling (DN) is considered to be a safe and effective method for reducing pain and improving muscle function, provoking a local contraction response in the muscle of myofascial trigger points.

Aims The main aim of this study was to evaluate the physiological changes that take place in the medial gastrocnemius (MG) with DN treatment using thermography.

Material and Methods In total, 20 basketball players participated in this study, aged between 21 and 39 years old. Initially, before performing DN, pre-intervention images were taken. Subsequently, DN was performed on the MG with the maximum of local twitch responses, evaluating both MG of both legs. Images were taken immediately after DN, and at 15 and at 30 minutes. For this study, a thermographic camera was used (FLIR TUR E60) and the images were analyzed using FLIR TOOLS software. For the DN technique, needles were used measuring $0.30 \times 40$. The variables studied were the maximum temperature $\left(T_{\max }\right)$, minimum temperature $\left(T_{\min }\right)$ and mean temperature $\left(T_{\text {avg }}\right)$, in degrees Celsius.

Results A decreased temperature exists $\left(T^{\circ}\right)$ both for $T_{\max }, T_{\min }$ and $T_{\text {avg }}$ before and after the DN intervention. We observed a significant difference $(p=0.035)$ between $\mathrm{T}_{\min }$ pre-intervention and immediately after the intervention in the left leg. The other significant difference found was between $\mathrm{T}_{\min }$ pre-intervention on the left leg and after 30 minutes $(p=0.009)$. We found significant differences between $T_{\min }$ pre and immediately post intervention on the left leg $(p=0.021), T_{\text {min }}$ pre and after 15 minutes
DOI https://doi.org/ $10.1055 / \mathrm{s}-0039-3402507$. ISSN 2386-4591.
Copyright $\odot 2019$ by Thieme Revinter Publicações Ltda, Rio de Janeiro, Brazil
License terms

(®) $\Theta \circledast$ 
on the left leg $(p=0.007)$ and pre intervention and after 30 minutes on the left leg $(p=0.002)$. Other significant differences on the right leg were $T_{\min }$ pre-intervention and immediately after $(p=0.019)$, and after 15 minutes $(p=0.008)$.

Conclusions Dry needling may be a good method for reducing inflammation of the trigger point for its ability to decrease temperature, and therefore may allow us to decrease the maximum, minimum and average temperature of the muscle belly. 